\title{
Right Atrial Tamponade Post Atrial Septal Defect Surgery in a Child
}

\section{Kumar V ${ }^{1 *}$, Tiwari $\mathbf{N}^{2}$, Joshi Sajan ${ }^{3}$ and Kumar $\mathbf{G}^{2}$}

${ }^{1}$ Pediatric Cardiologist, Army Hospital, R\&R, New delhi, India

${ }^{2}$ Pediatric Cardiothoracic surgeon, Army Hospital, R\&R, New delhi, India

${ }^{3}$ Cardio thoracic Anaesthetist, Army Hospital, R\&R, New delhi, India

\begin{abstract}
This report describes a rare and life threatening occurrence of right atrial tamponade secondary to massive intrapericardial clot compressing the right atrium, in a three year old child post atrial septal defect surgery.
\end{abstract}

Keywords: Atrial septal defect; Pericardial effusion

\section{Introduction}

Pericardial effusions are common after congenital heart surgery [1]. However massive clot compressing isolated right atrium causing tamponade and hemodynamic compromise is extremely rare. We present images of one such case occurring in a four year old girl child post Atrial Septal Defect (ASD) surgery.

\section{Case Report}

A 4-year-old girl child was diagnosed as a case of large ASD $(23 \mathrm{~mm})$ with deficient rims. She was referred for ASD closure. Her preoperative evaluation showed normal hemogram and biochemical parameters. Her total cardiopulmonary bypass time was fourty minutes and the surgery was uneventful. Chest was closed after achieving complete hemostasis, and a pericardial drainage was placed. She was kept on ventilator with plans to extubate next morning. After 12 hours of surgery she started having episodes of bradycardia with gradually decreasing blood pressure. An urgent transthoracic echocardiography was done which showed a massive $(4 \mathrm{~cm} \times 4 \mathrm{~cm})$ intrapericardial clot compressing the right atrium with minimal in flow through the tricuspid valve (Figure. 1). Additionally there was moderate left ventricle dysfunction. Hemoglobin was $11 \mathrm{gm} \%$ (immediate postop $14 \mathrm{gm} \%$ ) while platelet was marginally reduced to 96 thousand/cubic mm. Prothrombin time (PT) and Partial Thromboplastin Time with Kaolin (PTTK) were normal. Child was urgently taken up for reexploration and the clot was removed. Hemodynamic parameters improved dramatically with improvement in left ventricle function. Child recovered completely and was discharged after three days of observation.

\section{Discussion and Conclusion}

Pericardial effusions are common after congenital heart surgery [1]. Intrapericardial hematoma compressing isolated right atrium

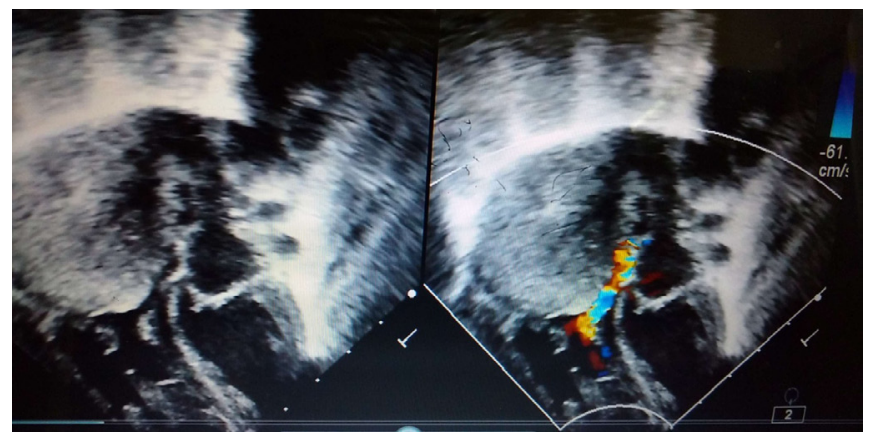

Figure 1: Showing large intrapericardial clot compressing the right atrium while colour doppler on right showing minimal inflow through tricuspid valve. causing tamponade and hemodynamic compromise is extremely rare. Henry et al. [2] have described one such case in an adult post mitral valve replacement eight days after surgery. The diagnosis was made on computed tomography scan. Another incidence was reported by Kochar et al. [3] thirty one days after aortic valve surgery in an adult. The latter had late postoperative leak. Our case is unique in that firstly it occurred few hours after surgery in a patient who was not on any antiplatelet or oral anticoagulants. Secondly patient had mediastinal drainage but this incidence happened, probably drain might have got blocked. This report highlights the clinical presentation of such a case and importance of quick reassessment by transthoracic echocardiography.

\section{References}

1. Cheung EWY, Ho SA, Tang KKY, Chau AKT, Chiu CSW, et al. (2003) Pericardial effusion after open heart surgery for congenital heart disease. Heart 89: $780-783$.

2. Huang HD, Garcia M, Alam M, Misra A, Lakkis N, et al. (2014) Post-operative intrapericardial hematoma presenting as isolated right atrial tamponade. J Cardiol Cases 9: 48-49.

3. Kochar GS, Jacobs LE, Kotler MN (1990) Right atrial compression in postoperative cardiac patients: detection by transesophageal echocardiography J Am Coll Cardiol 16: 511-516.
*Corresponding author: Kumar V, Pediatric Cardiologist, Army Hospital, R\&R, Dhaula kuan, New delhi, India, Tel: 7042743322; E-mail:Vk3532@gmail.com

Received June 22, 2017; Accepted June 30, 2017; Published July 04, 2017

Citation: Kumar V, Tiwari N, Sajan J, Kumar G (2017) Right Atrial Tamponade Post Atrial Septal Defect Surgery in a Child. J Cardiovasc Dis Diagn 5: i107. doi: 10.4172/2329-9517.1000i107

Copyright: @ 2017 Kumar V, et al. This is an open-access article distributed under the terms of the Creative Commons Attribution License, which permits unrestricted use, distribution, and reproduction in any medium, provided the original author and source are credited. 